\title{
Effects of exogenous lipids and cold acclimation on lycopene production and fatty acid composition in Blakeslea trispora
}

\author{
Feng Lingran ${ }^{1}$, Wang Qiang ${ }^{1 *}$, Yu Xiaobin ${ }^{2}$ and Fred Kwame ${ }^{2}$
}

\begin{abstract}
Exogenous lipids serving as stimulators to improve lycopene production in Blakeslea trispora have been widely reported. However, the selection basis of exogenous lipids and their effects on intracellular lipids are not very clear. In this study, five plant oils with different fatty acid compositions were selected to investigate their effects on lycopene production, fatty acid composition and the desaturation degree of intracellular lipids. Among the oils, soybean oil, with a fatty acid composition similar to that of mycelium, exhibited the best stimulating effect on lycopene formation (improvement of $82.1 \%$ ). The plant oils enhanced the total content of intracellular lipids and the desaturation degree of reserve lipids due to the alteration of fatty acid composition, especially in neutral lipids. Lycopene production was increased with the improved desaturation degree of intracellular lipids, which may be attributed to the enhancement of storage capacity for lycopene in storage lipid, thus reducing the feedback regulation of free lycopene. In addition, the increase of the desaturation degree of reserve lipids through temperature-changing fermentation also enhanced lycopene production. The present study could serve as a basis for a better understanding of the relationship between the fatty acid composition of reserve lipids and lycopene production.
\end{abstract}

Keywords: Lycopene, Cold acclimation, Exogenous lipids, Blakeslea trispora

\section{Introduction}

Lycopene, a member of the carotenoids, has been widely applied in the pharmaceutical, feed, and food industries (Feofilova et al. 2006; Nelis and Leenheer 1991). Increasing evidence supports the role of lycopene against some diseases, such as cardiovascular diseases and prostate cancer (Khachik et al. 2002; Story et al. 2010). Lycopene can be synthesized by chemical pathways, plants and microorganisms. Compared with chemical synthetic lycopene, natural-origin lycopene extracted from plants (mainly tomato) and microorganisms (mainly B. trispora) has a broader market due to the increasing consumer demand for "all natural" products, although synthetic and natural lycopene have identical bioavailability in humans (Hoppe et al. 2003; Papaioannou et al. 2016). The main

\footnotetext{
*Correspondence: wangqiang@htu.edu.cn

${ }^{1}$ College of Life Sciences, Henan Normal University, Xinxiang 453007,

China

Full list of author information is available at the end of the article
}

advantages of natural lycopene are more eco-friendly and safe to human compared with synthetic lycopene (Martínez-Cámara et al. 2018). Natural lycopene is approved for coloring matters within European Union and FDA, but chemical synthetic lycopene is not permitted. Moreover, the consumer perception that "natural is good" has been motivating the increase of natural lycopene markets. Although plant-origin lycopene still prevails in the current lycopene market, microbial lycopene has been commercialized by some companies employing the fungus $B$. trispora, such as Vitatene (Leon, Spain) and the North China Pharmaceutical Group (Shijiazhuang, China). Compared with plants, microorganisms have the advantages of a rapid growth rate, controllable conditions, and a high purity and percentage of lycopene in cells (LópezNieto et al. 2004). B. trispora, a zygomycota fungus with two mating types (termed 'plus' and 'minus'), occupies a prominent place among microbial producers of lycopene. Lycopene is an intermediate of the carotenoid biosynthetic pathway in B. trispora strains. To obtain lycopene, 
either a cyclase inhibitor, i.e., 2-methylimidazole (Pegklidou et al. 2008), or genetic means is required to prevent the formation of $\beta$-carotene and promote the accumulation of lycopene. Similar to plant origins, lycopene configurations in B. trispora mainly consist of all-trans forms (>90\%), although lycopene in vitro can isomerize to the mono or poly-cis form under heating or light (Authority 2005; Shi 2000).

The low yield of the final product is one of the main limitations of lycopene production by $B$. trispora. Numerous studies revealed that mutation breeding (Mehta et al. 2003; Rodriguez et al. 2009), various stimulants (Bhosale 2004; Shi et al. 2012; Wang et al. 2012), and process optimization (Mantzouridou et al. 2005, 2002; Mantzouridou and Naziri 2017) have been successfully used for enhancing lycopene production. Growth and lycopene synthesis are known to be stimulated by vegetable oils with high contents of linoleic and especially linolenic acids (Nanou and Roukas 2016; Sevgili and Erkmen 2019). The addition of plant oils improved the content of cellular unsaturated triacylglycerol in B. trispora compared with that obtained using glucose as the sole carbon source (Mantzouridou and Tsimidou 2007). Theoretically, exogenous lipids can be used for (a) synthesizing intracellular compounds and obtaining energy and (b) the biosynthesis of both lycopene and cellular lipids because they use acetyl-CoA (resulting from lipid degradation) as a common precursor substance. The catabolism of lipids promotes glucose towards the hexose monophosphate pathway (HMP) due to intracellular energy excess, hence increasing the production of NADPH. Thus, cellular lipid and lycopene synthesis can be stimulated because the biosynthesis and desaturases of both carotenoids and aliphatic chains require NADPH as co-factors (Beyer et al. 1994; Garba et al. 2017; Miziorko 2011). However, the effects exogenous lipids on intracellular lipids and the relationship between cellular fatty acid composition and lycopene production are not very clear.

Several modes of change of cellular fatty acids at varying environmental temperatures have been found in fungi, yeasts and bacteria, such as the degree of fatty acid unsaturation, fatty acid chain length, and cellular fatty acid content. The main change reflects the degree of unsaturation of fatty acyl chains through the control of fatty acid desaturation both at the level of transcription and post-transcriptional regulation (Dyer et al. 2001; Gargano et al. 1995; Vigh et al. 2005). Remodeling of membrane composition and functionality to maintain proper cellular function is widely accepted and termed homeoviscous adaptation (Cossins 1994). In addition to phospholipids, B. trispora also reserves large amounts of neutral lipids in lipid bodies where lipophilic carotenoids are deposited. It has been shown that hypothermic conditions can result in (a) an increase in the degree of desaturation of fatty acids in the lipid bilayer and (b) changes in the qualitative and quantitative composition of neutral lipids (Feofilova et al. 2000). Feofilova et al. (2005) showed that the degree of desaturation of fatty acids of both $(+)$ and $(-)$ strains of B. trispora increased in the lipid bilayer, although the (-) strain lacks linolenic acid.

Plant oils are known to improve the lycopene production and biomass of B. trispora. However, their effects on intracellular fatty acid composition and the selection basis of exogenous plant oils are not very clear. In this work, the lipid composition and lycopene production of B. trispora in response to different exogenous plant oils and temperature variation were investigated. The aims were to reveal the effects of several common plant oils on intracellular lipids and the relationship between cellular fatty acid composition and lycopene production. Based on these findings, lycopene production could be improved by altering the intracellular fatty acid composition of B. trispora, such as by adding suitable plant oils, changing the fermentation temperature, and adjusting other culture conditions.

\section{Materials and methods}

\section{Microorganisms and culture conditions}

The microorganisms used in this study were $B$. trispora NRRL 2895 mating type (+) and B. trispora I5 mating type $(-)$. The strain 15 , which presents a super yellow colony color and lovastatin resistance, was a high-yield lycopene mutant of $B$. trispora NRRL 2896 mating type (-) (Wang et al. 2013). The strains were grown on potato dextrose agar medium at $25{ }^{\circ} \mathrm{C}$ for 4 days and thereafter subcultured every 30 days. The spores obtained were suspended in $10 \mathrm{~mL}$ of sterile water to prepare the inocula. Spore suspensions containing $2.0 \times 10^{5}$ and $1.0 \times 10^{6}$ spores $/ \mathrm{mL}$ of the strains NRRL 2895 and 15 was transferred to $250-\mathrm{mL}$ conical flasks, each containing $50 \mathrm{~mL}$ of seed medium. The flasks were cultivated on a rotary shaker with an agitation rate of $200 \mathrm{rpm}$ at $25{ }^{\circ} \mathrm{C}$ for $48 \mathrm{~h}$. The seed medium $(\mathrm{g} / \mathrm{L})$ contained: corn starch 30, soybean meal 50, $\mathrm{KH}_{2} \mathrm{PO}_{4} 1.5, \mathrm{MgSO}_{4} \cdot 7 \mathrm{H}_{2} \mathrm{O} 0.5$ and thiamine $\mathrm{HCl} 0.002$, and the $\mathrm{pH}$ was adjusted to 6.5 . All medium components were sterilized at $121^{\circ} \mathrm{C}$ for $20 \mathrm{~min}$.

\section{Fermentation conditions}

Fermentation was carried out in $250-\mathrm{mL}$ flasks with $25 \mathrm{~mL}$ of media (three replicas of each treatment) and $10 \%(\mathrm{v} / \mathrm{v})$ inoculum containing a $1: 5(\mathrm{v} / \mathrm{v})$ mixture of each seed broth grown separately. The fermentation medium $(\mathrm{g} / \mathrm{L})$ contained: corn starch 50 , soybean meal 25, cottonseed oil 40, $\mathrm{KH}_{2} \mathrm{PO}_{4} 1.5, \mathrm{MgSO}_{4} \cdot 7 \mathrm{H}_{2} \mathrm{O} 0.5$ and thiamine $\cdot \mathrm{HCl} 0.002$, and the $\mathrm{pH}$ was adjusted to 6.5 . All 
medium components were sterilized at $121^{\circ} \mathrm{C}$ for $20 \mathrm{~min}$. The flasks were cultivated on a rotary shaker with an agitation rate of $200 \mathrm{rpm}$ at $25^{\circ} \mathrm{C}$. A cyclase inhibitor, i.e., 2-methylimidazole, was added at a concentration of $0.3 \mathrm{~g} / \mathrm{L}$ after $48 \mathrm{~h}$ of fermentation (Pegklidou et al. 2008). Cultures were maintained for $120 \mathrm{~h}$, and then the cells were harvested to determine the dry cell weight and lycopene content.

\section{Temperature-changing fermentation}

To evaluate the effect of different culture temperature on the growth of B. trispora, the inoculated flasks were cultivated at different constant temperature that as follows: $16,18,20,22,24,26$, and $28^{\circ} \mathrm{C}$. Cultures were maintained for $120 \mathrm{~h}$, and then the cells were harvested to determine the dry cell weight.

To obtain the time course of lycopene concentration and dry biomass by B. trispora under constant temperature and varying temperature, the experiments were designed as follows. 72 inoculated flasks were divided into two groups: constant temperature and varying temperature. During constant-temperature fermentation, the temperature was maintained at $25{ }^{\circ} \mathrm{C}$ until the end. During temperature-changing fermentation, the culture process was divided into three phases: phase I for the first $84 \mathrm{~h}$, phase II for the following $24 \mathrm{~h}$, and phase III for the last $36 \mathrm{~h}$. The temperatures were maintained at $25{ }^{\circ} \mathrm{C}$ for phase I and phase III, and $20{ }^{\circ} \mathrm{C}$ for phase II. Every $12 \mathrm{~h}$ interval, three flasks of each group were taken out to determine the lycopene concentration and dry cell weight. In addition, the samples collected from the varying temperature group at 72,96 and $120 \mathrm{~h}$ were simultaneously used to determine the fatty acid composition of intracellular lipid of B. trispora.

\section{Lipid and fatty acid analysis}

At appropriate time intervals, a $10-\mathrm{mL}$ sample was collected from the culture broth and then centrifuged at $10,000 \times g$ for $20 \mathrm{~min}$. The sediment was disrupted by freezing in liquid nitrogen and subsequently thawing and disintegrating with quartz sand. Lipid was extracted by using a chloroform-methanol (2:1) mixture that was stirred on a magnetic mixer. Lipid extracts were separated on thin-layer chromatography plates $(20 \times 20 \mathrm{~cm})$ coated with silica gel 60 , using solvents with different degrees of polarity (Nichols 1963). Neutral lipids (NL) were separated using the solvent system hexane-ether-acetic acid (85:15:1). Phospholipids (PL) were separated using two solvent systems successively in the same direction: hexane-ether-acetic acid (85:15:1) and chloroform-methanol-acetic acid-water (25:15:4:2). Glycolipids (GL) were separated using the solvent system chloroform-methanol-water (65:15:2). The lipid fractions containing NL, PL, GL were scraped off the plates for fatty acid analysis, respectively. Lipid classes were visualized by iodine vapor and identified by comparison of their $R_{f}$ values with those of known standards (Sigma). Individual lipid classes were relatively quantified by a densitometer. Fatty acid methyl esters (FAMEs) were prepared as described by Lepage and Roy (1984) and then analyzed using a gas chromatography mass spectrometry (Thermo Scientific TQ 8000, USA) equipped with a Supelco SPB-50 capillary column $(30 \mathrm{~m} \times 0.25 \mathrm{~mm}, 0.25 \mu \mathrm{m})$. The column temperature was programmed using the gradient mode starting at $110^{\circ} \mathrm{C}$ maintained for $3 \mathrm{~min}$, then gradually increasing to $220^{\circ} \mathrm{C}$ at a rate of $20^{\circ} \mathrm{C} \mathrm{min}{ }^{-1}$ and maintaining the latter temperature for $25 \mathrm{~min}$. The temperature of the injector and detector were maintained at 250 and $260{ }^{\circ} \mathrm{C}$, respectively. Samples $(1 \mu \mathrm{L})$ were injected with a split ratio of 50:1 by the autoinjector. Helium was used as a carrier gas. The ion source and ion source surface temperatures were set to $200{ }^{\circ} \mathrm{C}$ and $250{ }^{\circ} \mathrm{C}$, respectively. Electron impact ionization $(70 \mathrm{eV})$ in scan mode $(\mathrm{m} / \mathrm{z} 60-600)$ at a rate of 20 scans/s was used. Mass spectra of all detected compounds were compared with spectra in the NIST library 2.0 (2008 version) and the in-house mass spectra library database established by Umea Plant Science Center. Individual fatty acids were identified by comparing their retention times with those of known FAME standards (Sigma). Nonadecanoic acid (C19:0) was used as an internal standard. Fatty acid amounts were relatively quantified by calculating their chromatographic peak areas.

\section{Extraction and analysis of lycopene}

After $120 \mathrm{~h}$, a $10-\mathrm{mL}$ sample was collected from culture broth and then centrifuged at $10,000 \times g$ for $20 \mathrm{~min}$. The sediment was washed with distilled water and recentrifuged (triple). Dry biomass weight was determined after drying at $105^{\circ} \mathrm{C}$ overnight. To measure lycopene content, the sediment was dried in a vacuum drier at $40{ }^{\circ} \mathrm{C}$ and then crushed using mortar and pestle, followed by extraction of lycopene with petroleum ether. Repeated extractions of lycopene were carried out with the above solvent until the residue became colorless. Lycopene produced was analyzed by high-performance liquid chromatography (HPLC). The HPLC was equipped with a ZORBAX Eclipse Plus C18 column $(250 \mathrm{~mm} \times 4.6 \mathrm{~mm})$ at $30{ }^{\circ} \mathrm{C}$. The mobile phase was acetonitrile: acetone $(60: 40, \mathrm{v} / \mathrm{v})$ at a flow rate of $1 \mathrm{~mL} / \mathrm{min}$. The absorption of lycopene was measured at $470 \mathrm{~nm}$. Lycopene was identified by comparing with the retention time of lycopene standard (Sigma), 
and quantitative analysis was performed by the singlepoint calibration method.

\section{Statistical analysis}

The reported data are mean values of three independent experiments. Statistical differences between different treatments (groups) were determined by analysis of variance (ANOVA) and Tukey's post hoc test. Results were considered significant when differences had values of $\mathrm{p}<0.05$. Pearson's correlation was used to analyze the relationships between the solubility of lycopene and the desaturation degree of plant oils. All statistical analyses were performed with SPSS 18.0 (SPSS Inc., Chicago, Illinois).

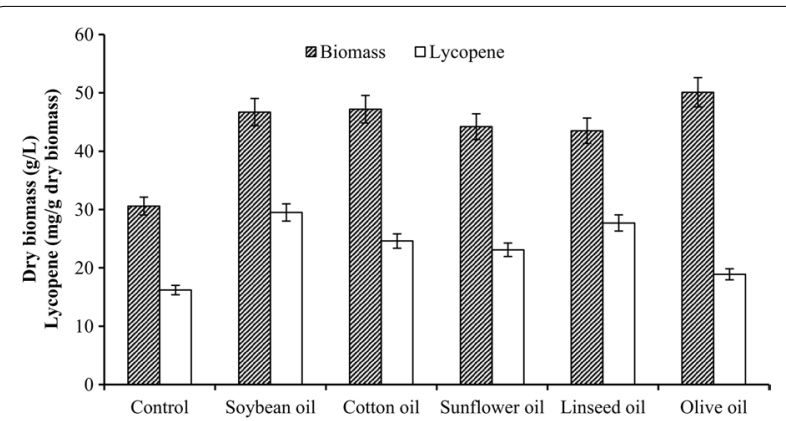

Fig. 1 Effect of different plant oils on dry biomass and lycopene production by $B$. trispora. Error bars indicate SEs of mean values. Black-striped bars, biomass; off-white-colored bars, lycopene

\section{Results}

Effect of exogenous lipids on dry biomass and lycopene production

According to the available literature, the lipids of $B$. trispora grown in media with glucose or corn flour predominantly contained palmitic $\left(\mathrm{C}_{16: 0}\right)$, stearic $\left(\mathrm{C}_{18: 0}\right)$, oleic $\left(\mathrm{C}_{18: 1}\right)$, linoleic $\left(\mathrm{C}_{18: 2}\right)$, and linolenic $\left(\mathrm{C}_{18: 3}\right)$ fatty acids (Tereshina et al. 2005, 2010; Vereschagina et al. 2010). When corn starch was used as the main carbon source, the effects of the oils used in this study on total lipids, dry biomass, and lycopene production are shown in Fig. 1. In the presence of oils, these three parameters were increased relative to those without oil. Soybean oil was more effective in stimulating lycopene formation $(40.1 \mathrm{mg} / \mathrm{g})$ than other oils $(\mathrm{p}<0.05$, ANOVA analysis and Tukey's post hoc test). The highest volumetric production $(1.3 \mathrm{~g} / \mathrm{L})$ was obtained in the presence of soybean oil ( $<<0.01$, ANOVA analysis and Tukey's post hoc test). The fatty acid compositions of the oils were determined by assessing fatty acid profiles (Table 1). These plant oils largely contained saturated palmitic and stearic fatty acids and unsaturated oleic, linoleic, and linolenic fatty acids. Among these oils, the highest contents of palmitic, oleic, linoleic, and linolenic fatty acids were found in cotton, olive, soybean, and linseed oils, respectively $(\mathrm{p}<0.05$, ANOVA analysis and Tukey's post hoc test). The contents of stearic acid in these oils were not significantly different ( $p>0.05$, ANOVA analysis and Tukey's post hoc test).

Table 1 Effect of plant oils on the fatty acid composition of intracellular lipids of $B$. trispora

\begin{tabular}{|c|c|c|c|c|c|c|c|c|c|c|c|c|}
\hline & \multicolumn{3}{|c|}{ Control } & \multicolumn{3}{|c|}{ Soybean oil } & \multicolumn{3}{|c|}{ Olive oil } & \multicolumn{3}{|c|}{ Linseed oil } \\
\hline & $\mathrm{NL}$ & PL & GL & NL & PL & $\mathrm{GL}$ & NL & PL & $\mathrm{GL}$ & $\overline{N L}$ & $\mathrm{PL}$ & $\mathrm{GL}$ \\
\hline$C_{14: 0}$ & $2.9^{\mathrm{a}}$ & 2.5 & 10.5 & 3.3 & 1.0 & 8.6 & 3.5 & 1.8 & 9.7 & 3.0 & 2.1 & 9.1 \\
\hline$C_{15: 0}$ & - & - & - & - & 0.4 & - & - & - & 0.6 & - & - & 0.5 \\
\hline$C_{16: 0}$ & 21.3 & 22.8 & 18.9 & 10.7 & 15.1 & 12.9 & 12.4 & 18.8 & 13.5 & 11.5 & 21.0 & 14.5 \\
\hline$C_{16: 1}$ & 0.3 & - & 0.3 & 0.5 & - & 0.5 & 0.4 & - & 0.6 & - & - & 0.4 \\
\hline$C_{17: 0}$ & - & 3.2 & - & 1.0 & 1.7 & - & - & 1.5 & - & - & 1.5 & - \\
\hline$C_{18: 0}$ & 15.1 & 5.0 & 19.5 & 6.2 & 3.3 & 14.8 & 8.0 & 3.8 & 12.7 & 8.7 & 4.7 & 14.3 \\
\hline$C_{18: 1}$ & 21.9 & 21.2 & 21.1 & 25.1 & 20.5 & 24.5 & 56.3 & 29.7 & 30.5 & 20.5 & 19.7 & 23.4 \\
\hline$C_{18: 2}$ & 34.3 & 44.8 & 27.2 & 46.5 & 47.3 & 33.0 & 12.4 & 42.9 & 26.3 & 26.3 & 45.9 & 30.2 \\
\hline$C_{18: 3}$ & 1.9 & 0.5 & - & 4.1 & 0.7 & - & 4.0 & 0.7 & - & 18.5 & 4.7 & 3.5 \\
\hline$C_{20: 0}$ & 0.8 & - & - & 0.5 & 0.4 & 0.6 & 0.7 & - & 0.5 & 0.7 & - & 0.4 \\
\hline$C_{20: 1}$ & 1.0 & - & 1.8 & 0.4 & - & 3.5 & 0.6 & - & 3.0 & - & - & 0.5 \\
\hline$C_{20: 2}$ & - & - & - & - & - & 0.4 & 1.0 & 0.8 & 1.7 & 0.5 & 0.4 & 1.5 \\
\hline$C_{20: 3}$ & - & - & - & - & - & - & - & - & - & - & - & 0.4 \\
\hline$C_{20: 4}$ & - & - & 0.4 & - & - & 0.3 & - & - & 0.5 & - & - & 0.3 \\
\hline$C_{22: 0}$ & 0.5 & - & 0.3 & 0.7 & - & 0.5 & 0.7 & - & - & - & - & 0.5 \\
\hline$C_{22: 1}$ & - & - & - & - & - & 0.4 & - & - & 0.4 & - & - & 0.5 \\
\hline $\begin{array}{l}\text { Desaturation } \\
\text { degree }\end{array}$ & 0.98 & 1.12 & 0.79 & 1.31 & 1.17 & 0.97 & 0.96 & 1.19 & 0.93 & 1.30 & 1.26 & 1.01 \\
\hline
\end{tabular}

a All value mean the relative content of fatty acids, $\%$ of lipid fraction 


\section{Effect of plant oils on fatty acid composition of cellular lipids}

To further investigate the influence of oil on lycopene synthesis, the fatty acid compositions of the NL, PL, and GL of the mycelium in the presence of different oils were assayed. Based on the composition analysis of plant oils, unsaturated fatty acids showed a positive influence on lycopene production. Three plant oils (soybean, linseed, and olive oils) with significantly different degrees of unsaturation were chosen for further analysis $(\mathrm{p}<0.01)$. Meanwhile, their effects on lycopene production also showed significant differences $(\mathrm{p}<0.01)$. The results are shown in Table 1 . The overall trend of fatty acid composition in the presence of oils was an increase in unsaturated fatty acids against a decrease in saturated fatty acids relative to the compositions without oil. This resulted in an increase in the desaturation degree of fatty acids of the NL, PL, and GL of the mycelium. Unlike the strong influence of soybean and linseed oils on the desaturation degree of fatty acids, olive oil was relatively weak. This may be attributed to the difference in the fatty acid compositions of these three oils. The relative contents of the lipid fractions and the total lipid contents in the mycelium in the presence of different oils are shown in Table 2. Both NL and total lipid were increased in the presence of oils compared with those without oil. The percentage increase of NL in lipid fractions was not measured against the background of the decreased absolute contents of PL and GL. This is due to the significant increase in the absolute content of NL compared with the slight increase in PL and GL.

\section{Solubility of lycopene in different plant oils}

The fatty acid compositions of intracellular lipid have some similarities with that of plant oils. Therefore, to explore the influence of the fatty acid composition of intracellular lipid on the solubility of lycopene in mycelium, the solubilities of lycopene in different plant oils were determined. As Fig. 2 shows, linseed oil exhibited the highest solubility value, which was two times higher than secondary soybean oil. Olive oil showed the lowest solubility value. In addition, there was a positive correlation between the solubility of lycopene and the desaturation degree of plant oils (pearson correlation, $R^{2}=0.972$, $\mathrm{p}<0.01)$.

\section{Effects of temperature variation on dry biomass and lycopene production}

It has been shown that cold acclimation can alter fatty acid composition in intracellular lipids, especially the degree of unsaturation of fatty acyl chains. Therefore, the effects of temperature variation on lycopene production and fatty acid composition were investigated, with the aim of providing support for the correlation between lycopene production and the desaturation degree of intracellular lipids. As shown in Fig. 3, the growth of B. trispora was

Table 2 Effect of culture temperature on fatty acid composition of intracellular lipid of $B$. trispora

\begin{tabular}{|c|c|c|c|c|c|c|c|c|c|}
\hline & \multicolumn{3}{|c|}{ Phase $\mathrm{I}^{\mathrm{a}}$} & \multicolumn{3}{|c|}{ Phase II } & \multicolumn{3}{|c|}{ Phase III } \\
\hline & NL & $\mathrm{PL}$ & GL & NL & PL & GL & NL & PL & GL \\
\hline$C_{14: 0}$ & $3.5^{b}$ & 2.5 & 8.7 & 1.2 & 0.5 & 6.1 & 3.0 & 2.7 & 7.2 \\
\hline$C_{15: 0}$ & - & - & - & - & - & 0.5 & - & 0.3 & 0.3 \\
\hline$C_{16: 0}$ & 15.4 & 20.8 & 18.5 & 3.0 & 7.8 & 7.2 & 7.2 & 18.4 & 17.1 \\
\hline$C_{16: 1}$ & 0.5 & - & 0.3 & 0.6 & - & - & 0.7 & - & - \\
\hline$C_{17: 0}$ & 1.2 & 3.2 & - & 0.8 & 0.4 & 0.4 & 0.8 & 3.0 & 0.7 \\
\hline$C_{18: 0}$ & 18.2 & 4.7 & 11.2 & 9.0 & 2.5 & 9.1 & 12.8 & 5.8 & 11.1 \\
\hline$C_{18: 1}$ & 23.8 & 21.5 & 24.0 & 32.2 & 33.2 & 31.2 & 24.5 & 20.5 & 26.4 \\
\hline$C_{18: 2}$ & 33.2 & 46.8 & 33.6 & 48.4 & 55.2 & 37.9 & 43.2 & 47.6 & 35.4 \\
\hline$C_{18: 3}$ & 2.4 & 0.5 & - & 3.0 & 4.6 & - & 6.3 & 1.5 & 0.5 \\
\hline$C_{20: 0}$ & 0.5 & - & 0.3 & 0.7 & 0.4 & 0.5 & 0.5 & 0.2 & 0.3 \\
\hline$C_{20: 1}$ & 0.4 & - & 3.2 & - & - & 4.6 & - & - & - \\
\hline$C_{20: 2}$ & - & - & 0.4 & 0.3 & - & - & 0.3 & - & - \\
\hline$C_{20: 3}$ & - & - & 0.3 & - & - & 0.2 & - & - & 0.4 \\
\hline$C_{20: 4}$ & - & - & 0.6 & - & 0.4 & 1.1 & - & - & 0.3 \\
\hline$C_{22: 0}$ & 0.9 & - & 0.5 & 0.8 & - & 0.3 & 0.7 & - & - \\
\hline$C_{22: 1}$ & - & - & 0.4 & - & - & 0.9 & - & - & 0.3 \\
\hline $\begin{array}{l}\text { Desaturation } \\
\text { degree }\end{array}$ & 0.98 & 1.17 & 0.99 & 1.39 & 1.59 & 1.18 & 1.31 & 1.20 & 1.01 \\
\hline
\end{tabular}

\footnotetext{
a Sampling time of Phase I, Phase II and Phase III were 72, 96, and $120 \mathrm{~h}$, respectively
}

b All value mean the relative content of fatty acids, $\%$ of lipid fraction 


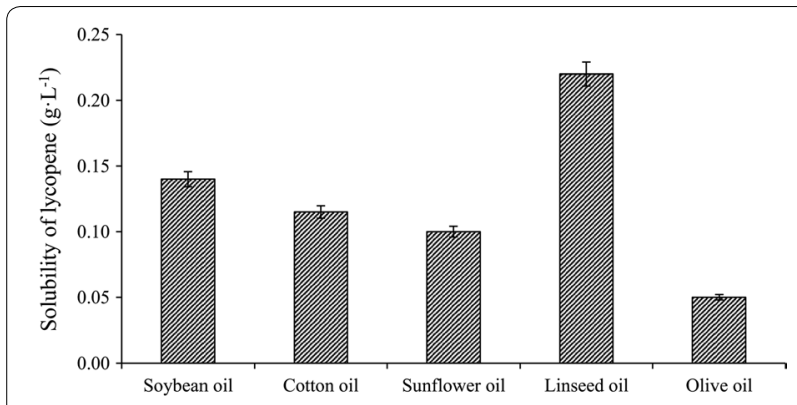

Fig. 2 Solubility of lycopene in different plant oils. Error bars indicate SEs of mean values

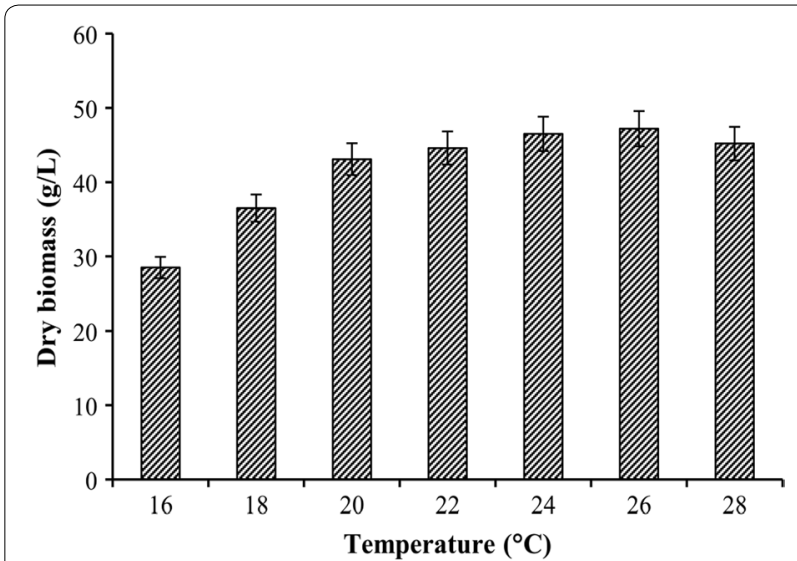

Fig. 3 Effect of different culture temperature on dry biomass by $B$. trispora. Error bars indicate SEs of mean values

obviously restricted when the culture temperature was below $20^{\circ} \mathrm{C}$. Hence, the cold stress temperature was chosen as $20^{\circ} \mathrm{C}$. The time courses of dry biomass and lycopene production under a suitable growth temperature are shown in Fig. 4a. The biomass increased rapidly at $12 \mathrm{~h}$, reached the maximum value at $48 \mathrm{~h}$, and remained almost constant afterwards. Lycopene production increased rapidly after adding cyclase inhibitor at $48 \mathrm{~h}$ and increased slowly after $84 \mathrm{~h}$. Figure $4 \mathrm{~b}$ shows the changes of the dry biomass and lycopene production with fermentation time under temperature variation. From Fig. 4a, the time of culture temperature adjustment to $20{ }^{\circ} \mathrm{C}$ ranged from 84 to $108 \mathrm{~h}$. The change in biomass under this variation was similar to that under constant temperature. Although lycopene concentration increased slightly under cold stress, it increased rapidly when the culture temperature was set to $25^{\circ} \mathrm{C}$ again. The final lycopene concentration reached $35.2 \pm 1.1 \mathrm{mg} / \mathrm{g}$ dry biomass under varying culture temperatures, which was $24 \%$ higher than that under constant temperature. The optimum temperature for lycopene production is not $20^{\circ} \mathrm{C}$, since the lycopene concentration remained almost constant at this temperature.
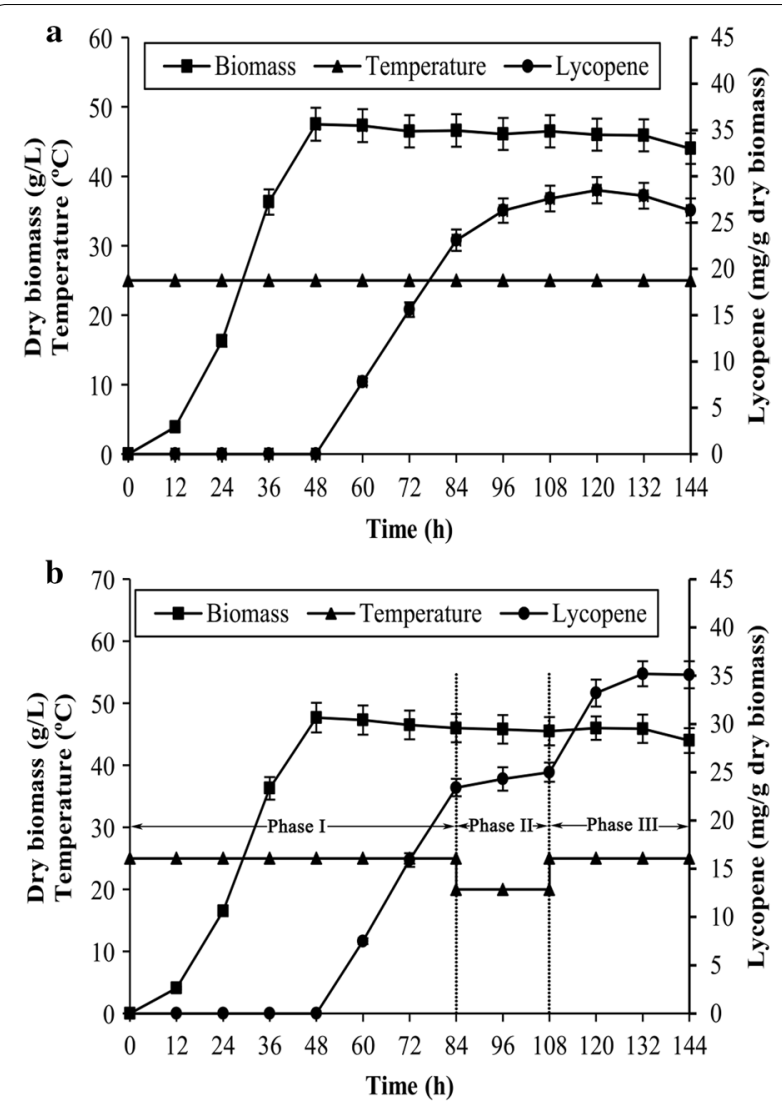

Fig. 4 Time course of lycopene concentration and dry biomass by B. trispora under a constant temperature and $\mathbf{b}$ varying temperature. Error bars indicate SEs of mean values. Solid square, dry biomass; solid triangle, temperature; solid circles, lycopene concentration

\section{Changes in fatty acid composition in cellular lipids under temperature variation}

The fermentation process was segmented into three phases based on temperature change. The fatty acid compositions of NL, PL, and GL during these three phases are shown in Table 3. When fermentation was carried out to phase two, the fatty acid composition exhibited

Table 3 Effect of plant oils on the composition and content of intracellular lipid and NADPH concentration by $B$. trispora

\begin{tabular}{|c|c|c|c|c|c|}
\hline & \multicolumn{3}{|c|}{$\begin{array}{l}\text { Lipid fractions, } \\
\% \text { of total lipids }\end{array}$} & \multirow{2}{*}{$\begin{array}{l}\text { Total content } \\
\text { of lipids, } \% \text { of dry } \\
\text { biomass }\end{array}$} & \multirow{2}{*}{$\begin{array}{l}\text { NADPH } \\
\text { (nmol/mg } \\
\text { protein) }\end{array}$} \\
\hline & NL & PL & $\mathrm{GL}$ & & \\
\hline Control & 80.4 & 15.0 & 4.6 & $15.3 \pm 0.6$ & $0.42 \pm 0.01$ \\
\hline Soybean oil & 92.5 & 5.6 & 1.9 & $45.3 \pm 2.1$ & $1.1 \pm 0.01$ \\
\hline Olive oil & 92.5 & 5.8 & 1.7 & $43.1 \pm 2.3$ & $0.93 \pm 0.02$ \\
\hline Linseed oil & 89.0 & 8.5 & 2.5 & $31.2 \pm 1.4$ & $1.02 \pm 0.01$ \\
\hline
\end{tabular}

Data of absolute content are expressed as the mean \pm SD from three replications 
considerable changes due to the influence of the hypothermic conditions. The main modulations involved the increase of unsaturated oleic and linoleic fatty acids against the background of the decrease in saturated stearic and palmitic fatty acids. During phase three, the fatty acid composition of PL and GL almost returned to that in phase one. Surprisingly, the fatty acid composition of NL remained similar to that in phase two; i.e., NL contained high contents of unsaturated oleic and linoleic fatty acids, although culture temperature was recovered. Hence, the desaturated degree of fatty acids of NL increased and persisted until phase three.

\section{Discussion}

Exogenous plant oils are hydrolyzed to fatty acids and glycerol by fungal exolipases. Fatty acids can (a) be oxidized to acetyl-CoA by $\beta$-oxidation and (b) be incorporated into reserve lipid structures, such as lipid bodies (Čertík et al. 1997). Acetyl-CoA not only provides energy for metabolism through the tricarboxylic acid cycle but also serves as a common precursor of carotenoids and lipids. Consequently, the increase of precursor stimulates the formation of downstream products. Lycopene is a highly unsaturated hydrocarbon that contains 11 conjugated and two unconjugated double bonds (Shi and Maguer 2000). The desaturation degree of lycopene is much higher than that of intracellular lipids or plant oils. Thus, the increase in the desaturation degree of intracellular lipids was favorable for the dissolution of lycopene. The direct incorporation of exogenous fatty acids, especially linoleic and linolenic acids, enhances the desaturation degree of the fatty acids of the acylglycerols, thus increasing the solubility of lycopene (Mantzouridou and Tsimidou 2007). Therefore, this promotes the storage of large amounts of lycopene in the lipid bodies, which mainly consist of acylglycerols (Danilova and Tereshina 2019; Walther and Farese Jr 2012). NADPH is an essential cofactor in both the elongation and desaturation of the carbon chain of lycopene. One mole of produced lycopene requires $16 \mathrm{~mol}$ of NADPH and $24 \mathrm{~mol}$ of ATP (data not shown). In oil-enriched substrates, intracellular energy is excessive, resulting from the catabolism of fatty acid-based substrates. Hence, more glucose is metabolized through the HMP pathway, thereby increasing the production of NADPH (Reshamwala and Modi 1985). This, together with the reduced demand for NADPH, ATP and carbon in fatty acid biosynthesis due to the direct incorporation of extracellular unsaturated aliphatic chains, results in the enhanced supply of NADPH, ATP and carbon for carotenoid synthesis.

Among the NL, PL, and GL, the fatty acids of the NL showed the most significant changes in response to different oils. In contrast, the fatty acids that prevailed in the oils were not significantly accumulated in the PL and GL of the mycelium. The GL acts as a recognition site for specific chemicals as well as helps to maintain the stability of the membrane (Schnaar 2004). PL is the major component of the cell membrane and can provide membrane fluidity and mechanical strength (Bloom et al. 1991). Owing to these special functions, the fatty acid components of the GL and PL were steady. NL has a role in energy and lipophilic substance storage (Athenstaedt 2010). Its content is large and variable. The highest fatty acid components of soybean, olive, and linseed oils are linoleic $\left(\mathrm{C}_{18: 2}\right)$, oleic $\left(\mathrm{C}_{18: 1}\right)$, and linolenic $\left(\mathrm{C}_{18: 3}\right)$ fatty acid, respectively. These acids significantly accumulated in the NL of the mycelium owing to the addition of these three oils. The desaturation degree of fatty acids of NL decreased slightly, although the absolute content of unsaturated oleic acid increased with olive oil addition. This is because the relative contents of linoleic, linolenic and other unsaturated acids decreased with the increase in the absolute content of oleic acid. Calculation of the desaturation degree was based on the relative contents of these fatty acids. The uptake of oils can not only enhance the content of NL of the mycelium but also increase the desaturation degree of fatty acids of the NL (Mantzouridou and Tsimidou 2007). Therefore, more lycopene can be deposited in storage lipid thus reducing the feedback inhibition of free lycopene. Among these oils, soybean oil exhibited the best stimulating effect on lycopene production. In addition to the above mechanism, soybean oil contains high levels of lecithin, a very effective emulsifier of lycopene that can facilitate its transfer from one enzyme to the next.

Lycopene is a secondary metabolite and not necessary for growth in B. trispora. Microbes reduce or even suspend unimportant secondary metabolism to maintain basic functions under cold stress (Feofilova 2003). Energy and building material used for lycopene biosynthesis may be reduced under cold stress, resulting in the deceleration of lycopene biosynthesis (phase II in Fig. 4b). But lycopene concentration increased rapidly after returning to normal culture temperature as expected (phase III). The reason for this phenomenon may be the change of lycopene solubility depending on the alteration of fatty acid composition in the reserve lipids. Hence, the changes in fatty acid composition in cellular lipids at varying temperatures were investigated. Microbes respond to temperature stress by changing the composition of the membrane and reserve lipids (Beney and Gervais 2001; Zhu et al. 2007). The changes that occur in lipid acyl chains mainly include saturation and desaturation, isomerization, and changes in the length of fatty acid carbon 
chains (Suutari and Laakso 1994; Wagenen et al. 2012). The data obtained enabled us to hypothesize that the changes in the fatty acid composition of NL are difficult to recover, for a time at least. The enhancement of lycopene under cold stress can be mainly attributed to the increase of the desaturation degree of NL. Combined with the influence of plant oils on lycopene production and the fatty acid composition of intracellular lipids, it can be concluded that the increase in the desaturation degree of intracellular lipids benefits the accumulation of lycopene in B. trispora.

The oils with fatty acid compositions similar to that of the fungus had more advantages in altering intracellular fatty acid composition and enhancing lycopene production relative to other oils. To our knowledge, this is the first study on the effect of cold acclimation on lycopene biosynthesis by $B$. trispora. Lycopene production were substantially improved just changing fermentation temperature, which may bring some commercial interests. In addition to cold acclimation, other conditions, such as oxygen concentration and $\mathrm{pH}$, can also affect fatty acid composition and lipid content. Therefore, these conditions could be experimentally determined to enhance lycopene production in future studies.

\section{Abbreviations \\ HMP: hexose monophosphate pathway; NL: neutral lipids; PL: phospholipids; GL: glycolipids; FAMEs: fatty acid methyl esters; HPLC: high-performance liquid chromatograph.}

\section{Acknowledgements}

Not applicable.

\section{Authors' contributions}

$F L, W Q$ and $Y X$ designed the entire research project. FL carried out the experiments, analyzed and interpreted the data, and drafted the article. FK reviewed the article and checked the writing in English. All authors read and approved the final manuscript.

\section{Funding}

This work was supported by the Training Program of the National Natural Science Foundation of China [Grant Number 5101049170806].

\section{Availability of data and materials}

Not applicable

\section{Ethics approval and consent to participate}

Not applicable as this article does not contain any studies with human participants or animals by any of the authors.

\section{Consent for publication}

Not applicable.

\section{Competing interests}

The authors declare that they have no competing interests.

\section{Author details}

${ }^{1}$ College of Life Sciences, Henan Normal University, Xinxiang 453007, China.

${ }^{2}$ The Key Laboratory of Industrial Biotechnology, Ministry of Education, and School of Biotechnology, Jiangnan University, Wuxi 214122, China.
Received: 6 June 2019 Accepted: 1 October 2019

Published online: 11 October 2019

\section{References}

Athenstaedt K (2010) Neutral lipids in yeast: synthesis, storage and degradation. Handbook of hydrocarbon and lipid microbiology, pp 471-480

Authority EFS (2005) Opinion of the scientific panel on dietetic products, nutrition and allergies on a request from the commission related to an application on the use of a-tocopherol-containing oil suspension of lycopene from Blakeslea trispora as a novel food ingredient. EFSA J 212:1-29

Beney L, Gervais P (2001) Influence of the fluidity of the membrane on the response of microorganisms to environmental stresses. Appl Microbiol Biotechnol 57(1-2):34-42

Beyer P, Nievelstein V, Albabili S, Bonk M, Kleinig H (1994) Biochemical aspects of carotene desaturation and cyclization in chromoplast membranes from Narcissus pseudonarcissus. Pure Appl Chem 66(5):1047-1056

Bhosale P (2004) Environmental and cultural stimulants in the production of carotenoids from microorganisms. Appl Microbiol Biotechnol 63(4):351361. https://doi.org/10.1007/s00253-003-1441-1

Bloom M, Evans E, Mouritsen OG (1991) Physical properties of the fluid lipidbilayer component of cell membranes: a perspective. Q Rev Biophys 24(3):293-397

Čertík M, Balteszov L, Šajbidor J (1997) Lipid formation and $\gamma$-linolenic acid production by Mucorales fungi grown on sunflower oil. Lett Appl Microbiol 25(2):101-105

Cossins A (1994) Homeoviscous adaptation of biological membranes and its functional significance. Temperature adaptation of biological membranes. Portland Press, London, pp 63-76

Danilova OA, Tereshina VM (2019) Effect of trisporoids on lipogenesis of the $\mathrm{T}(-)$ strain of a mycelial fungus Blakeslea trispora. Microbiology 88(1):118-119. https://doi.org/10.1134/s0026261719010028

Dyer JM, Chapital DC, Cary JW, Pepperman AB (2001) Chilling-sensitive, post-transcriptional regulation of a plant fatty acid desaturase expressed in Yeast. Biochem Biophys Res Commun 282(4):1019-1025. https://doi. org/10.1006/bbrc.2001.4667

Feofilova E (2003) Deceleration of vital activity as a universal biochemical mechanism ensuring adaptation of microorganisms to stress factors: a review. Appl Biochem Microbiol 39(1):1-18

Feofilova E, Tereshina V, Memorskaya A, Khokhlova N (2000) Different mechanisms of the biochemical adaptation of mycelial fungi to temperature stress: changes in the lipid composition. Microbiology 69(5):509-515

Feofilova E, Tereshina V, Memorskaya A (2005) Biochemical mechanisms of temperature adaptation in the $(+)$ and $(-)$ strains of Blakeslea trispora. Microbiology 74(6):650-654

Feofilova EP, Tereshina VM, Memorskaya AS, Dul'kin LM, Goncharov NG (2006) Fungal lycopene: the biotechnology of its production and prospects for its application in medicine. Microbiol Immunol 75(6):629-633

Garba L, Ali MSM, Oslan SN, Rahman RNZRBA (2017) Review on fatty acid desaturases and their roles in temperature acclimatisation. J Appl Sci 17(6):282-295

Gargano S, Dilallo G, Kobayashi GS, Maresca B (1995) A temperature-sensitive strain of Histoplasma capsulatum has an altered $\Delta 9$-fatty acid desaturase geneacid desaturase gene. Lipids 30(10):899-906

Hoppe PP, Krämer K, Berg HVD, Steenge G, Vliet TV (2003) Synthetic and tomato-based lycopene have identical bioavailability in humans. Eur J Nutr 42(5):272-278

Khachik F, Carvalho L, Bernstein PS, Muir GJ, Zhao DY, Katz NB (2002) Chemistry, distribution, and metabolism of tomato carotenoids and their impact on human health. Exp Biol Med 227(10):845

Lepage G, Roy CC (1984) Improved recovery of fatty acid through direct transesterification without prior extraction or purification. J Lipid Res 25(12):1391-1396

López-Nieto MJ, Costa J, Peiro E, Méndez E, Rodríguez-Sáiz M, Fuente JLDL, Cabri W, Barredo JL (2004) Biotechnological lycopene production by mated fermentation of Blakeslea trispora. Appl Microbiol Biotechnol 66(2):153-159

Mantzouridou FT, Naziri E (2017) Scale translation from shaken to diffused bubble aerated systems for lycopene production by Blakeslea 
trispora under stimulated conditions. Appl Microbiol Biotechnol 101(5):1845-1856

Mantzouridou F, Tsimidou MZ (2007) Carotenoid pattern in Blakeslea trispora grown on oil-enriched substrates with regard to triacylglycerol species accumulation. Eur J Lipid Sci Technol 109(1):3-10. https://doi. org/10.1002/ejlt.200600197

Mantzouridou F, Roukas T, Kotzekidou P (2002) Effect of the aeration rate and agitation speed on beta-carotene production and morphology of Blakeslea trispora in a stirred tank reactor: mathematical modeling. Biochem Eng J 10(2):123-135

Mantzouridou F, Roukas T, Achatz B (2005) Effect of oxygen transfer rate on $\beta$-carotene production from synthetic medium by Blakeslea trispora in shake flask culture. Enzym Microb Technol 37(7):687-694. https://doi. org/10.1016/j.enzmictec.2005.02.020

Martínez-Cámara S, Rubio S, Río HD, Rodríguez-Sáiz M, Barredo JL (2018) Lycopene Production by Mated Fermentation of Blakeslea trispora, Microbial Carotenoids. Humana Press, New York, pp 257-268

Mehta BJ, Obraztsova IN, Cerda-Olmedo E (2003) Mutants and intersexual heterokaryons of Blakeslea trispora for production of $\beta$-carotene and lycopene. Appl Environ Microbiol 69(7):4043-4048. https://doi.org/10.1128/ aem.69.7.4043-4048.2003

Miziorko HM (2011) Enzymes of the mevalonate pathway of isoprenoid biosynthesis. Arch Biochem Biophys 505(2):131-143

Nanou K, Roukas T (2016) Waste cooking oil: a new substrate for carotene production by Blakeslea trispora in submerged fermentation: biomass, bioenergy, biowastes, conversion technologies, biotransformations, production technologies. Biores Technol 203:198-203

Nelis HJ, Leenheer APD (1991) Microbial sources of carotenoid pigments used in foods and feeds. J Appl Bacteriol 70(3):181-191

Nichols BW (1963) Separation of the lipids of photosynthetic tissues: improvements in analysis by thin-layer chromatography. Biochim et Biophys acta (BBA) 70:417-422

Papaioannou EH, Liakopoulou-Kyriakides M, Karabelas AJ (2016) Natural origin lycopene and its "green" downstream processing. Crit Rev Food Sci Nutr 56(4):686

Pegklidou K, Mantzouridou F, Tsimidou MZ (2008) Lycopene production using Blakeslea trispora in the presence of 2-methyl imidazole: yield, selectivity, and safety aspects. J Agric Food Chem 56(12):4482-4490

Reshamwala P, Modi V (1985) Increased levels of lipids, NAD-isocitrate dehydrogenase and glucose-6-phosphate dehydrogenase and phosphatases in mated cultures of Blakeslea trispora. Indian J Exp Biol 23:327-329

Rodriguez M, Teresa A, De E, Antonio C, Costa Perez J, Ruiz O, Antonio M, Fraile Yecora N, De La FMJL, Rodriguez Saiz M (2009) Improved method of production of lycopene by the fermentation of selected strains of Blakeslea trispora. European Patent 1471151B1, 4 Nov 2009

Schnaar RL (2004) Glycolipid-mediated cell-cell recognition in inflammation and nerve regeneration. Arch Biochem Biophys 426(2):163-172
Sevgili A, Erkmen O (2019) Improved lycopene production from different substrates by mated fermentation of Blakeslea trispora. Foods 8(4):120

Shi J (2000) Lycopene in tomatoes: chemical and physical properties affected by food processing. Crit Rev Biotechnol 20(4):293-334

Shi J, Maguer ML (2000) Lycopene in tomatoes: chemical and physical properties affected by food processing. Crit Rev Food Sci Nutr 40(1):1-42

Shi Y-q, Xin X-I, Yuan Q-p (2012) Improved lycopene production by Blakeslea trispora with isopentenyl compounds and metabolic precursors. Biotechnol Lett 34(5):849-852

Story EN, Kopec RE, Schwartz SJ, Harris GK (2010) An update on the health effects of tomato lycopene. Annu Rev Food Sci Technol 1(1):189-210

Suutari M, Laakso S (1994) Microbial fatty acids and thermal adaptation. Crit Rev Microbiol 20(4):285-328

Tereshina V, Memorskaya A, Feofilova E (2005) Lipid composition of cells of heterothallic strains in the developmental cycle of Blakeslea trispora. Appl Biochem Microbiol 41(4):394-398

Tereshina V, Memorskaya A, Feofilova E (2010) Lipid composition of the mucoraceous fungus Blakeslea trispora under lycopene formation-stimulating conditions. Microbiology 79(1):34-39

Vereschagina O, Memorskaya A, Tereshina $\mathrm{V}$ (2010) The role of exogenous lipids in lycopene synthesis in the mucoraceous fungus Blakeslea trispora. Microbiology 79(5):593-601

Vigh L, Escribá PV, Sonnleitner A, Sonnleitner M, Piotto S, Maresca B, Horváth I, Harwood JL (2005) The significance of lipid composition for membrane activity: new concepts and ways of assessing function. Prog Lipid Res 44(5):303-344. https://doi.org/10.1016/j.plipres.2005.08.001

Wagenen V, Jon Miller, Tyler W, Hobbs Paul, Crowe Braden (2012) Effects of light and temperature on fatty acid production in Nannochloropsis Salina. Energies 5(3):731-740

Walther TC, Farese RV Jr (2012) Lipid droplets and cellular lipid metabolism Annu Rev Biochem 81:687-714

Wang JF, Liu XJ, Liu RS, Li HM, Tang YJ (2012) Optimization of the mated fermentation process for the production of lycopene by Blakeslea trispora NRRL 2895 (+) and NRRL 2896 (-). Bioprocess Biosyst Eng 35(4):553-564. https://doi.org/10.1007/s00449-011-0628-6

Wang Q, Luo W, Gu Q-y, Feng L-r, Li H-g, Yu X-b (2013) Enhanced lycopene content in Blakeslea trispora by effective mutation-screening method. Appl Biochem Biotechnol 171(7):1692-1700

Zhu L, Zhang X, Ji L, Song X, Kuang C (2007) Changes of lipid content and fatty acid composition of Schizochytrium limacinum in response to different temperatures and salinities. Process Biochem 42(2):210-214

\section{Publisher's Note}

Springer Nature remains neutral with regard to jurisdictional claims in published maps and institutional affiliations.

\section{Submit your manuscript to a SpringerOpen ${ }^{\circ}$ journal and benefit from:}

- Convenient online submission

- Rigorous peer review

- Open access: articles freely available online

- High visibility within the field

Retaining the copyright to your article

Submit your next manuscript at springeropen.com 